\title{
Double-Layer Silicon PIN Photodiode X-Ray Detector for a Future X-ray Timing Mission
}

\author{
Hua Feng ${ }^{\text {a,* }}$, Philip Kaaret ${ }^{\text {a }}$, Hans Andersson ${ }^{\text {b }}$ \\ a Department of Physics and Astronomy, University of Iowa, Van Allen Hall, Iowa \\ City, IA 52242 \\ ${ }^{\mathrm{b}}$ Oxford Instruments Analytical Oy, P.O. Box 85, 02631 Espoo, Finland
}

\begin{abstract}
A double-layer silicon detector consisting of two $500 \mu \mathrm{m}$-thick silicon PIN photodiodes with independent readouts was mounted in a vacuum chamber and tested with X-ray sources. The detector is sensitive from $1-30 \mathrm{keV}$ with an effective area of $6 \mathrm{~mm}^{2}$. The detector performs best at $-35{ }^{\circ} \mathrm{C}$ with an energy resolution of $220 \mathrm{eV}$ (FWHM, full width at half maximum) at $5.9 \mathrm{keV}$, and is able to operate at room temperature, $+25{ }^{\circ} \mathrm{C}$, with moderate resolution around $760 \mathrm{eV}$ (FWHM). The response of the top layer sensor is highly uniform across the sensitive area. This large-format silicon detector is appropriate for future X-ray timing missions.
\end{abstract}

Key words: X-ray, Si, PIN, timing

\section{Introduction}

New detectors with large effective areas, rapid timing response, and good sensitivity up to $\sim 30 \mathrm{keV}$ are required to fulfill the scientific goals for a future X-ray timing observatory [1] beyond the Rossi X-ray Timing Explorer (RXTE) [2]. RXTE has been operating for ten years with fruitful scientific results obtained from its Proportional Counter Array (PCA), which is close to the end of its lifetime. Thick silicon detectors are, perhaps, the best candidate detectors for future timing missions. Silicon PIN photodiodes are simple to fabricate, with mature applications in industry and science [3], and could be manufactured in large volumes at moderate cost with existing technology.

\footnotetext{
* Corresponding author.

Email address: hua-feng@uiowa.edu (Hua Feng).
} 
Large-format thick silicon PIN diode arrays sensitive in 1-30 keV would give spectral resolutions of a few hundred $\mathrm{eV}$ (FWHM) at $6 \mathrm{keV}$, which is better than that of PCA. Also, solid state detectors should have good long-term stability.

This paper describes the structure and test of a double-layer silicon PIN detector. We present measurement of the spectral resolution and its variation with temperature, and the uniformity of the detector efficiency, charge collection, and resolution. We conclude with a summary of the performance of the detector and the important characteristics for its application in X-ray astronomy.

\section{Detector and Experiment Configuration}

A double-layer silicon detector was assembled from two square silicon chips with high voltage applied to both chips on the middle layer between the chips and guard rings on both chips that create a circular effective area with a diameter of $2.75 \mathrm{~mm}$. This silicon detector sandwich is attached to an alumina substrate along with two FETs acting as the front stage of the detector preamplifiers. A peltier cooler (MI2012T-01AC from Marlow Industries, Inc.) is adhered on the other side of the alumina substrate using silver epoxy. The warm side of the cooler is connected to a copper heat sink which is mounted in the center of a CF-100 flange. Thin wires connected to the detector to pass high voltage and electronic signals and another two, thicker, wires to power the cooler are connected via feedthroughs in the flange to an electronics box mounted outside the vacuum regions and containing two preamplifiers and high voltage filtering circuitry.

The preamplifier is charge sensitive with a reset switch, instead of a feedback resistor, to discharge the feedback capacitor. The two preamplifier outputs for both layers are connected to shaping amplifiers with a gain of 1000 and adjustable shaping time. The shaped outputs are stretched to a pulse duration of $5 \mu \mathrm{s}$ and the stretched outputs are fed into a Wavebook (Wavebook 512A from IOtech, Inc.) for pulse digitization. The shaped pulse from each channel is fed into a single channel analyzer used to trigger the readout. A pulse above threshold from either channel will trigger the Wavebook to sample the pulses from both channels.

The CF-100 flange with the detector is mounted on the side of a vacuum chamber. An X-ray tube is located on the other side of the vacuum chamber on the same axis as the detector. A steel mask with a $0.33 \mathrm{~mm}$ diameter central hole is placed in front of the detector as a collimator. It is carried by a two dimensional linear stage in order to move the exposure point at different 
positions above the sensitive area. Radioactive source of ${ }^{55} \mathrm{Fe}$ and ${ }^{109} \mathrm{Cd}$ are mounted on the same mask and may be positioned in front of the detector as required.

\section{Spectral Resolution}

We obtained ${ }^{55} \mathrm{Fe}$ spectra at various temperatures in the range -35 to $+25^{\circ} \mathrm{C}$ with shaping times of $4 \mu \mathrm{s}$ and $16 \mu \mathrm{s}$. The energy resolution versus temperature is shown in Fig. 1. At high temperatures, a smaller shaping time such as 1 $\mu$ s gives better resolution, but also cause oscillations in the shaping amplifier output, which produces much more noise at low energies. At low temperature around $-35{ }^{\circ} \mathrm{C}$, a shaping time of $16 \mu$ s produces the best resolution, which, though, is not strongly influenced by changing the shaping time in the range 8-24 $\mu$ s.

Fig. 1 shows that the best resolution is obtained at $-35{ }^{\circ} \mathrm{C}$ with the shaping time of $16 \mu \mathrm{s}$. A moderate resolution of $763 \mathrm{eV}$ is obtained at temperature of $25^{\circ} \mathrm{C}$ with a shaping time of $4 \mu \mathrm{s}$. These two spectra are shown in Fig. 2. We fitted each spectrum with a model consisting of the sum of two Gaussian functions, corresponding to the $\mathrm{Mn} \mathrm{K} \alpha(5.9 \mathrm{keV})$ and $\mathrm{K} \beta(6.5 \mathrm{keV})$ lines respectively. This model provided an adequate fit to all of the spectra.

In an early measurement there appeared a notable condensation problem, which made the resolution become worse unexpectedly at temperatures below $-20{ }^{\circ} \mathrm{C}$. To reduce the condensation, we added another cooler inside the chamber and made it below $-40{ }^{\circ} \mathrm{C}$ for several days prior to the measurement. This helped much but would not prevent condensation if the detector stayed at low temperature longer than one hour. Therefore, every spectrum below 0 ${ }^{\circ} \mathrm{C}$ in Fig. 1 \& 2 was obtained when the detector was cooled down directly from room temperature within one hour. While spectra from Fig. 3 to Fig. 5 were obtained when the temperature stayed at $-20{ }^{\circ} \mathrm{C}$ for long time since those results did not suffer from the condensation problem much.

The electronic noise [4] can be described as

$$
\Delta E(e V)=2.355 \frac{w}{q}\left(c_{0} q I_{L}+c_{1} 2 k T C_{i n}^{2} / g_{m}+c_{2} A_{1 / f}+c_{3} C_{i n}^{2}\right)^{\frac{1}{2}}
$$

where $w$ is $3.6 \mathrm{eV} / \mathrm{e}-\mathrm{h}$ for silicon, $q$ is the electron charge, $I_{L}$ is the leakage current, $k$ is the Boltzmann constant, $T$ is the temperature, $C_{i n}$ is the total input capacitance, $g_{m}$ is the trasconductance of the input FET, $A_{1 / f}$ is the excess $1 / f$ noise, $c_{0-3}$ are coefficients for different noise power terms, $c_{0}$ is proportional to the shaping time and $c_{1}$ is inversely proportional to the shaping 
time. With a given shaping time, $c_{2} A_{1 / f}+c_{3} C_{i n}^{2}$ is a constant and the leakage current can be expressed as a exponential function. Therefore Eq. (1) can be re-written as

$$
\Delta E(e V)=2.355 \frac{w}{q}\left(a_{0} \mathrm{e}^{a_{1} T}+a_{2} T+a_{3}\right)^{\frac{1}{2}}
$$

where $a_{0-3}$ are constants.

We fit the two curves in Fig. 1 corresponding to the two different shaping times to Eq. (2) and obtained two sets of coefficients $a_{0-3}$. We find that the ratio $a_{0,16 \mu \mathrm{s}} / a_{0,4 \mu \mathrm{s}}=4.4$, which is close to the ratio of the two shaping times as expected. The coefficient $a_{1}$ is primarily determined by the temperature dependence of the leakage current. For a shaping time of $4 \mu \mathrm{s}$, we find $a_{1,4 \mu \mathrm{s}}=$ 0.11 corresponding to an increase in leakage current by a factor of 1.1 for an increase in temperature of $1^{\circ} \mathrm{C}$. This is consistent with our direct measurement of the temperature dependence of the leakage current and is close to that expected for silicon and measured for other Si PIN photodiodes. For a shaping time of $16 \mu \mathrm{s}$, we find $a_{1,16 \mu \mathrm{s}}=0.14$ which indicates a somewhat faster increase in the noise versus temperature. The determination of the linear component, i.e. $a_{2}$, is of high uncertainty, because the resolution tends to be a constant at low temperatures. The fitting results do not change significantly whether we force $a_{2}=0$ or not.

Applying a temperature of $-20{ }^{\circ} \mathrm{C}$ and a shaping time of $16 \mu$ s, we obtained spectra using the radioactive sources ${ }^{55} \mathrm{Fe}$ and ${ }^{109} \mathrm{Cd}$ and using an X-ray tube with Mo target. The ${ }^{55} \mathrm{Fe}$ radioactive source shows $\mathrm{Mn} \mathrm{K} \alpha$ and $\mathrm{K} \beta$ lines only on the top layer. The ${ }^{109} \mathrm{Cd}$ radioactive source presents $\mathrm{Ag} \mathrm{K} \alpha$ and $\mathrm{K} \beta$ lines on both layers and a $\mathrm{Si} \mathrm{K} \alpha$ line on the top layer. The latter is caused by X-rays striking the Si outside the sensitive region and producing a fluorescence X-ray which then interacts within the sensitive region. The X-ray tube was operated at voltage of $40 \mathrm{kV}$ and produces bremsstrahlung radiation and the characteristic emission lines from the Mo target and a $\mathrm{Cu}$ tube holding the window. In the bottom layer spectrum, there are strong $\mathrm{Au}$ lines from fluorescence in a Au strip located between the two detector layers. This line can also be seen in the top layer spectrum, but is not as prominent as in the bottom layer due to the high flux from the bremsstrahlung spectrum. The Ag lines in the tube spectrum arise from the silver epoxy centered and beneath the sandwich. Al lines from the bottom layer spectrum are X-rays reprocessing on the alumina substrate beneath the detector. The measured energy spectra are shown in Fig. 3 for the top layer with three sources and for the bottom layer with the X-ray tube only. There are 13 lines detected which are labeled on Fig. 3 and their energies and widths are listed in Table 1. 


\section{Uniformity}

To test the uniformity at different spots on the silicon detector, we placed a stainless steel mask with a $0.33 \mathrm{~mm}$ diameter pinhole in front of the detector and illuminated it using the X-ray tube to create a collimated beam. The mask was mounted on a 2-dimensional linear stage enabling us to scan the pinhole across the silicon surface. We selected 37 points evenly spaced by $0.4 \mathrm{~mm}$ to cover the sensitive area of the detector (see the top left panel of Fig. 4). The solid circle indicates the sensitive area and the dashed circle encircles points collimating X-rays all inside the sensitive area, e.g. the full response region.

The efficiency is measured as the total counting rate in $3-30 \mathrm{keV}$ band. An alternative measurement using only the characteristic emission lines from the tube produced the same result. The 2-dimensional efficiencies for both layers are plotted on the two bottom panels of Fig. 4. The top player is highly uniform. The bottom layer has a central area with low efficiency, which is caused by the $\mathrm{Au}$ high voltage strip which was incorrectly placed into the sensitive area in the assembly. The efficiency versus offset to the center is plotted in the top right panel of Fig. 4. A $2 \%$ systematic error is added to account for the instability of the X-ray tube flux.

We also measured the uniformity of charge collection and energy resolution. In top panel of Fig. 5, the Mo K $\alpha$ peak channels are measured at different spots on the detector for both top and bottom layer. The shift of the peak channel reflects the charge collection efficiency. For both layers, it is shown that the shift is within 5 channels, corresponding to $70 \mathrm{eV}$ for the $17.44 \mathrm{keV}$ peak. The variation is less than $0.5 \%$ and is much smaller than the FWHM. The variation of the resolution is within $20 \mathrm{eV}$ except one point in the bottom layer which is nearly $40 \mathrm{eV}$. These changes are very small indicating excellent uniformity of charge collection and energy resolution.

\section{Discussion and conclusion}

We have demonstrated a large-format silicon PIN photodiode which presents good performance in sensitivity, spectroscopy and timing. The detector has high quantum efficiency from 1-30 keV, fine spectral resolution of $220 \mathrm{eV}$ at $-35{ }^{\circ} \mathrm{C}$ and $760 \mathrm{eV}$ at $25{ }^{\circ} \mathrm{C}$, and good time resolution of several tens of microseconds or better.

The intended purpose of the double layer structure was to test background

rejection by coincidence in both layers. Spectra taken using a ${ }^{60}$ Co source to simulate the radiation environment on-orbit show that only a small fraction of 
the background events deposit energy on both layers simultaneously. However, the $\mathrm{Au}$ foil incorrectly inserted between the two detector layers, which gave bad uniformity for the bottom layer, decreased the efficiency for capturing coincident events. The on-orbit background counting rate for Si detectors and means for its reduction are an important topic for future study.

The top layer of the detector shows a excellent uniformity of efficiency, charge collection and spectral resolution. This suggests that we would obtain similar excellent uniformity for Si PIN photodiode detectors consisting of arrays of small pixels and operated as a position sensitive detector.

\section{Acknowledgements}

HF and PK thank The University of Iowa for support.

\section{References}

[1] P. Kaaret, in: X-Ray Timing 2003: Rossi and Beyond, AIP Conference, vol. 714, 2004, p. 423.

[2] H.V. Bradt, R.E. Rothschild, J.H. Swank, A\&AS 97 (1993) 355.

[3] K. Mitsuda, H. Kunieda, H. Inoue, R. Kelley, in: Proceedings of the SPIE, vol. 5488, 2004, p. 177

[4] J.S. Iwanczyk, B.E. Patt, in: Semiconductors for Room Temperature Nuclear Detector Applications: Electronics for X-ray and Gamma Ray Spectrometers, Academic Press, New York, 1995 


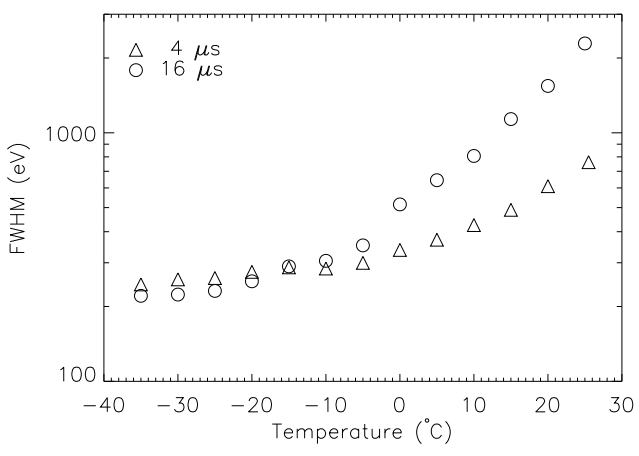

Fig. 1. Energy resolution (FWHM) vs. temperature for ${ }^{55} \mathrm{Fe} \mathrm{K} \alpha$ line with the shaping time of $4 \mu \mathrm{s}$ and $16 \mu$ s respectively.
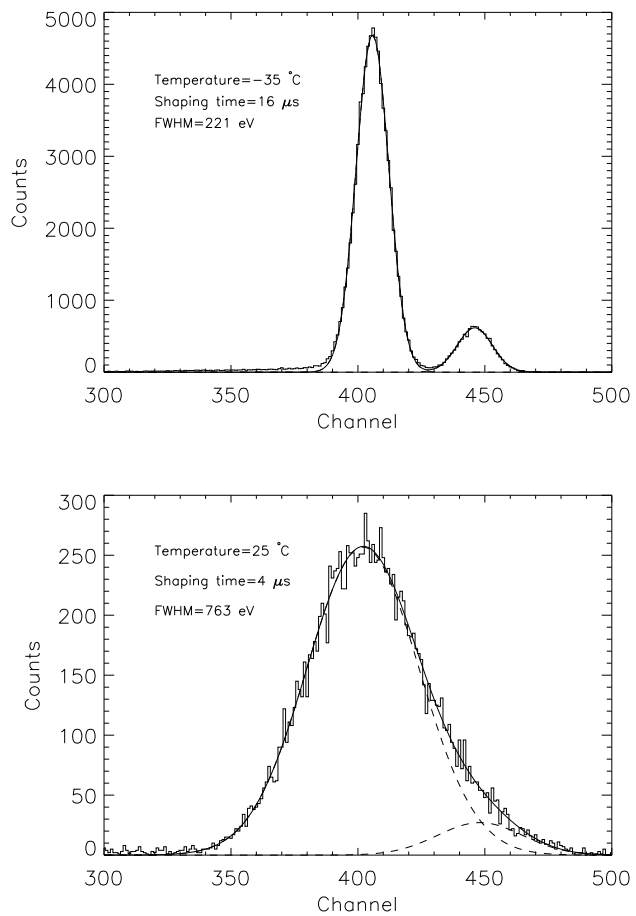

Fig. 2. Energy spectra of ${ }^{55} \mathrm{Fe}$. The best fitted spectrum with two Gaussian functions is plotted with dashed lines representing the $\mathrm{Mn} \mathrm{K} \alpha$ and $\mathrm{K} \beta$ lines respectively. Top: best performance at temperature of $-35{ }^{\circ} \mathrm{C}$ and shaping time of $16 \mu \mathrm{s}$. Bottom: at room temperature with the shaping time of $4 \mu \mathrm{s}$. 

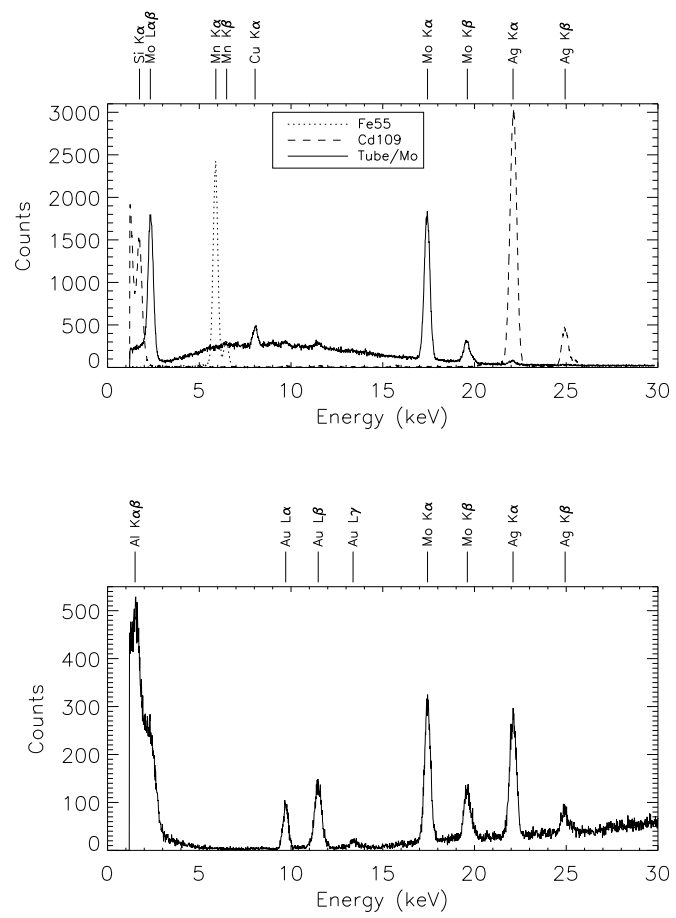

Fig. 3. Energy Spectra from ${ }^{55} \mathrm{Fe},{ }^{109} \mathrm{Cd}$ and the X-ray tube with Mo as the target. Emission lines are labeled on the top. Line energies and widths are listed in Table 1. Top: Top layer spectra; Bottom: Bottom layer spectrum from the X-ray tube only.

Table 1: Detected line energies and widths for top and bottom layers.

\begin{tabular}{rccccccc}
\hline \hline & $\mathrm{Al} \mathrm{K} \alpha \beta$ & $\mathrm{Si} \mathrm{K} \alpha$ & $\mathrm{Mo} \mathrm{L} \alpha \beta$ & $\mathrm{Mn} \mathrm{K} \alpha$ & $\mathrm{Mn} \mathrm{K} \beta$ & $\mathrm{Cu} \mathrm{K} \alpha$ & \\
\hline Energy (keV) & 1.5 & 1.74 & 2.23 & 5.90 & 6.49 & 8.04 & \\
Top (Fwhm, eV) & $\ldots$ & $\ldots$ & $\ldots$ & 282 & $\ldots$ & $\ldots$ & \\
Bottom (Fwhm, eV) & $\ldots$ & $\ldots$ & $\ldots$ & $\ldots$ & $\ldots$ & $\ldots$ & \\
\hline & $\mathrm{Au} \mathrm{L} \alpha$ & $\mathrm{Au} \mathrm{L} \beta$ & $\mathrm{Au} \mathrm{L \gamma}$ & $\mathrm{Mo} \mathrm{K} \alpha$ & $\mathrm{Mo} \mathrm{K} \beta$ & $\mathrm{Ag} \mathrm{K} \alpha$ & $\mathrm{Ag} \mathrm{K} \beta$ \\
energy (keV) & 9.71 & 11.48 & 13.38 & 17.44 & 19.61 & 22.10 & 24.94 \\
top (Fwнм, eV) & $\ldots$ & $\ldots$ & $\ldots$ & 359 & $\ldots$ & 424 & $\ldots$ \\
bottom (ғшнм, eV) & $\ldots$ & $\ldots$ & $\ldots$ & 376 & $\ldots$ & 447 & $\ldots$ \\
\hline
\end{tabular}



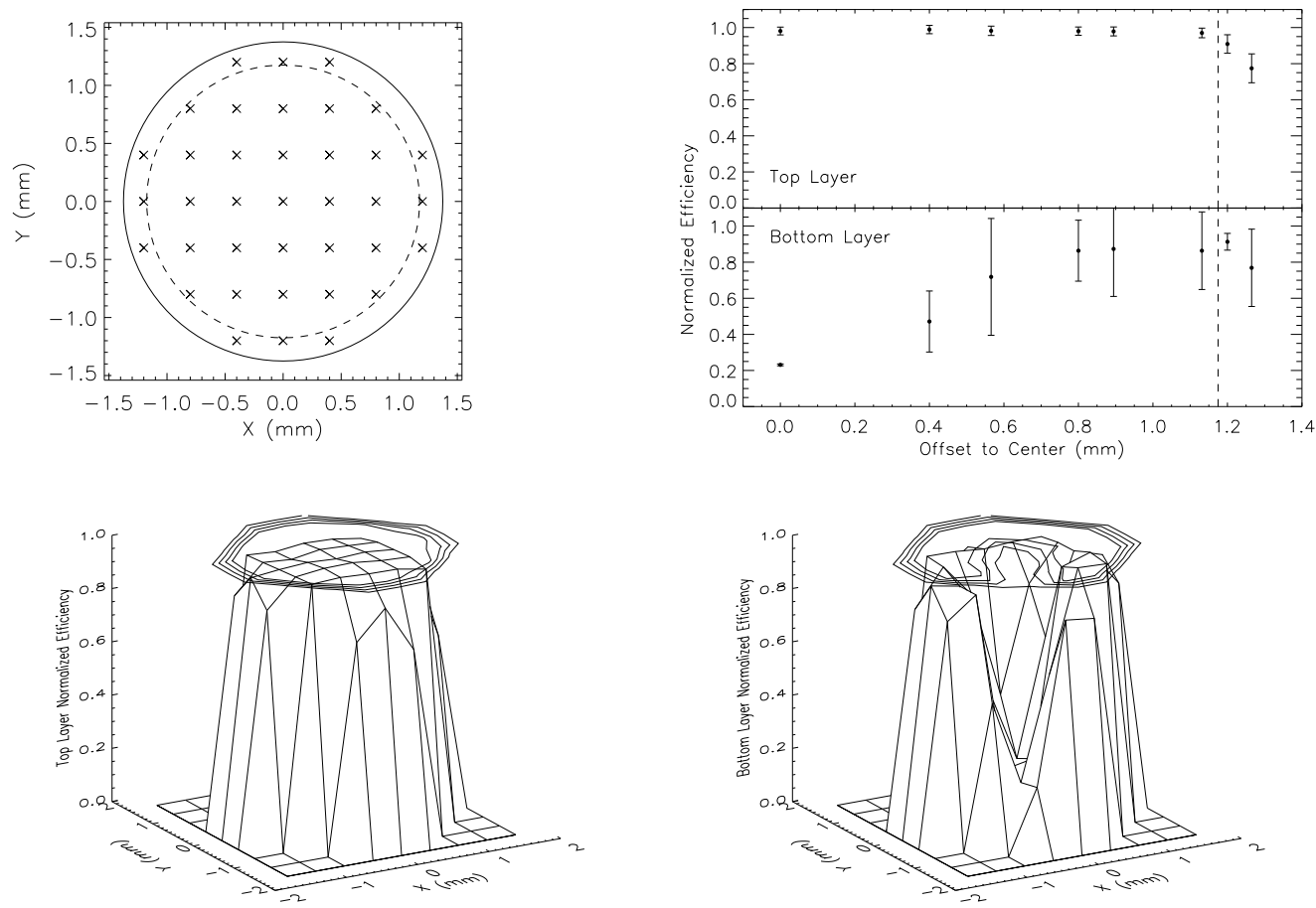

Fig. 4. Uniformity of efficiency at different points across the sensitive area. Top Left: crosses are positions from which spectra are taken from the X-ray tube. The solid circle is the sensitive area of the detector and the dashed line encircles full response points. Top Right: local efficiency vs. offset to the center. The dashed line indicates the full response radius. Bottom Left: local efficiency at different spots for the top layer. Bottom Right: local efficiency at different spots for the bottom layer. 

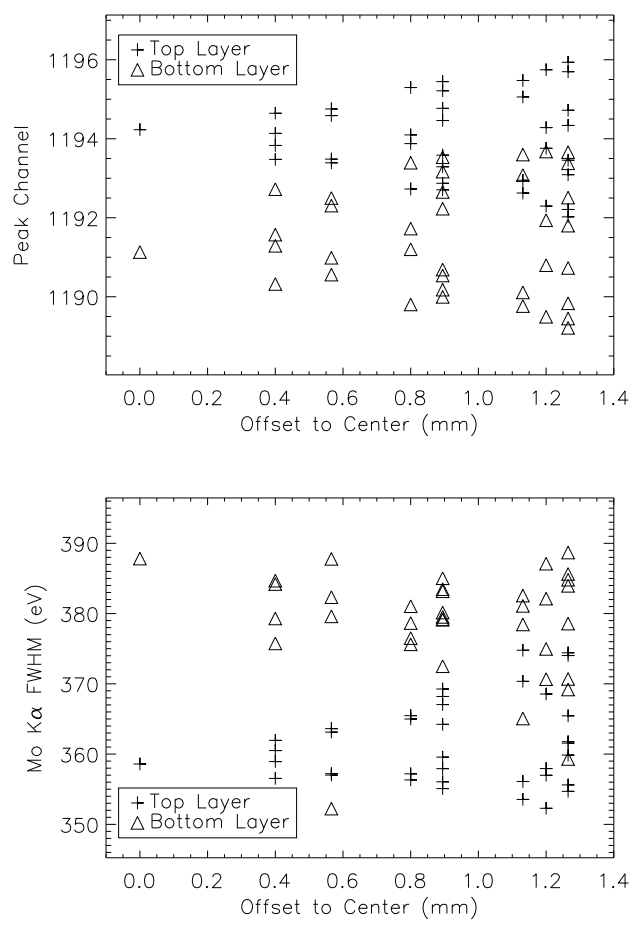

Fig. 5. Mo K $\alpha$ line peak channel (top panel) and FWHM (bottom panel) at different spots across the detector for both layers. 